\title{
Are environmental and behavioral interventions effective in reducing physical activity limitation and preventing falls in the visually impaired older individuals? - A Cochrane Review summary with commentary
}

\author{
Dilsad Sindel $(0$, Aydan 0ral $(1)$ \\ Department of Physical Medicine and Rehabilitation, Istanbul University Istanbul Faculty of Medicine, Istanbul, Turkey
}

Received: April 29, 2021 Accepted: April 30, 2021 Published online: May 25, 2021

The aim of this commentary is to discuss in a rehabilitation perspective the published Cochrane Review "Environmental and behavioural interventions for reducing physical activity limitation and preventing falls in older people with visual impairment"[1] by E, Li, Mclnally, Thomson, Shahani, Gray, Howe, Skelton ${ }^{a}$ under the direct supervision of Cochrane Eyes and Vision Group. This Cochrane Corner is produced in agreement with the Turkish Journal of Physical Medicine and Rehabilitation by Cochrane Rehabilitation.

Background: Visual impairment is a common health problem all over the World. ${ }^{[2]}$ According to the data of the Lancet Global Eye Health Commission, an estimated 596 million people worldwide experienced distant vision impairment in 2020, of which 43 million were identified as blind. 510 million people had uncorrected near vision impairment, due to lack of reading glasses. By 2050, due to the aging of the population and increased urbanization, the number of people with distant vision disorders are estimated to reach 895 million, 61 million of whom are expected to be blind. ${ }^{[3]}$ Increasing age increases visual impairment, which will contribute to the decrease in physical activity, increase in risk of falls and fractures. Also, mental health can be affected by aging with visual impairment, which increased the risk of dementia and cognitive decline, and probability of traffic accidents and mortality. Collectively, all these are likely to lead to greater needs for healthcare utility and cost. ${ }^{[2,3]}$ Falls and related injuries are particularly common in people over the age of 65 years. Approximately a third of elderly people fall at least once a year. ${ }^{[4]}$

Studies show that falls are an important cause of disability and death worldwide. The Global Burden of Disease Study provides age-standardized incidence of falls as 2238 per 100,000 in 2017. ${ }^{[5]}$

Early diagnosis and treatment of visual impairments can make it possible to decrease lack of mobility and the frequency of falls in advanced ages. In addition to person-related factors, multiple home hazards may be associated with risk of falls suggesting that there is a need for adapting homes for safety. ${ }^{[6]}$ A Cochrane review looked at evidence if addressing home hazards as well as behaviours were effective interventions for preventing falls and also for increasing physical activity in visually impaired older people..$^{[1]}$

Corresponding author: Dilsad Sindel, MD. İstanbul Üniversitesi İstanbul Tıp Fakültesi, Fiziksel Tıp ve Rehabilitasyon Anabilim Dalı, 34093 Fatih, İstanbul, Türkiye. e-mail: dilsadsindel@gmail.com 


\section{Environmental and behavioural interventions for reducing physical activity limitation and preventing falls in older people with visual impairment (E et al., 2020) $)^{[1]}$}

\section{What is the aim of this Cochrane review?}

The aim of this Cochrane Review is to determine the effectiveness in environmental and behavioral interventions which are used to prevent falls and reduce physical activity limitation in visually impaired older people.

\section{What was studied in the Cochrane review?}

The population addressed in this review was older people (aged 60 years and above) with irreversible visual impairment such as reduced visual field, low visual acuity, and poor contrast sensitivity and depth perception who were living independently or in residential settings. Older people with additional disabilities including hearing loss, neurological or cognitive or musculoskeletal impairments were not excluded. The interventions studied were environmental and/or behavioral interventions which included home safety modifications, minimizing home hazards, advice on behaviors to improve safety when performing activities of daily living (ADL), adaptive or assistive equipment provision, cognitive and other behavioral therapies, and low vision devices for visual rehabilitation, while other vision-correction interventions such as corrective lenses or cataract surgery were excluded. The interventions were compared to social/home visits or usual activity. The primary outcomes studied included physical activity as measured using continuous objective or self-reported measures such as body-fixed sensor activity monitoring or validated questionnaires or dichotomous selfreported measures, or falls as measured recording information on falls such as number of fallers and rate of falls. Secondary outcomes studied included fear of falling, quality of life, and fall-related beliefs, behaviors, and attitudes.

\section{Search methodology and up-to-dateness of the Cochrane review?}

The review authors searched for studies that had been published up to February 4, 2020 in electronic databases including Cochrane Central Register of Controlled Trials, MEDLINE and Embase Ovid, Cumulative Index to Nursing and Allied Health, Allied and Complementary Medicine Database, OT Seeker, metaRegister of Controlled Trials, International Standard Research Clinical Trial Number registry US National Institutes of Health Ongoing Trials Register
ClinicalTrials.gov, and World Health Organization (WHO) International Clinical Trials Registry Platform.

What are the main results of the Cochrane review?

The review included six randomized controlled trials involving 686 individuals, who were mostly female (69\%) visually impaired and physically independent elderly with a mean age of 80 years living mostly in their homes. Environmental interventions used in studies included home safety modification by occupational therapists. Behavioral interventions were mainly exercise including Exercise programs for fall management, Otago exercise program, Alexander technique, and Ashtanga-based yoga therapy. The comparator of social/home visits, included social support provided by volunteers in-home visits in the form of discussion of general topics about lifestyle while not giving clinical advice. The follow-up period in studies ranged from 2 to 12 months. No metaanalysis was possible due to the heterogeneity of the characteristics such as interventions and/or outcomes in the studies.

The review shows the results of single studies as the following:

When comparing environmental interventions with social/home visits

- No evidence of effect of the interventions on either physical activity as measured using step count, walking time and self-reported physical activity or the proportion of fallers and falls rate, moderate injurious falls, and serious injurious falls per person-year (1 study with 28 older people)

- No evidence of an effect on fear of falling and quality of life (1 study with 28 older people)

- A small benefit of home safety modifications over social/home visits on reducing falls in general and injurious falls as well (1 study with 196 older people)

When comparing behavioral interventions with usual activity or social/home visits

- No evidence of benefit of a Falls Management Exercise Program versus usual activity on physical activity (1 study with 59 older people)

- No evidence of benefit of the Otago exercise program combined with a standard osteoporosis exercise program versus standard osteoporosis exercise program alone in terms of the proportion of those who fell and time till the first fall occurred (1 study with 41 older people) 
- No evidence of a difference in effect between Otago Exercise Program plus vitamin D and social/home visits (1 study with 193 older people) and Alexander technique (1 study with 120 older people) and usual activity on falls in general and injurious falls

- No improvement in fear of falling with Falls Management Program (1 study with 59 older people) or Alexander technique (1 study with 114 older people), or Ashtanga-based yoga therapy (1 study with 21 older people) in comparison to usual care/usual activity

When comparing environmental interventions (home safety modification) with behavioral interventions (Otago Exercise Program)

- Evidence of effectiveness in reducing injurious falls (albeit not all falls) in favor of home safety modification (1 study with 197 older people)

When comparing environmental and behavioral interventions with social/home visits

- No evidence of a difference in effect between home hazards modification + Otago exercise program and social home visits in terms of either physical activity measurements or fear of falling (1 study with 28 older people)

- No difference in effect between the combined and single intervention with regard to proportion of fallers, falls rate, and moderate and severe injurious falls per person-year (1 study with 28 older people) and also in terms of falls in general and injurious falls (1 study with 194 older people)

When comparing environmental and behavioral interventions with environmental interventions

- No evidence of a difference in effect between home hazards modification and the Otago exercise program and only home hazard modification in terms of either physical activity as measured using step counts, walking time per minute, and self-reported activity (1 study, 30 older people) and falls as measured using proportion of fallers and falls rate, moderate injurious falls, and serious injurious falls per person-year (1 study with 198 older people).

- Evidence of effectiveness in reducing injurious falls (albeit not all falls) in favor of home safety modification with Otago Exercise Program (1 study with 197 older people).
- No evidence of a difference in effect between the combined and single intervention on fear of falling and quality of life (one study with 30 older people)

When comparing environmental and behavioral interventions in combination with only behavioral intervention

- No evidence of differing effects of the combined intervention over the single intervention on either proportion of fallers or injurious fallers (1 study with 195 older people)

To note, the evidence for all outcomes mentioned above was judged as low certainty evidence.

\section{How did the authors conclude?}

The authors concluded that there is an evidence gap regarding the effectiveness of the majority of environmental or behavioral interventions evaluated on increasing physical activity or preventing falls in the visually impaired elderly except for a small benefit on fall prevention that could possibly be provided by home safety modification delivered by occupational therapists in comparison to social/home visits based on low certainty evidence. In conclusion, current evidence cannot suggest that visually impaired older people can benefit from the most of the environmental and/or behavioral interventions designed to reduce their activity limitation and to make their movement safer while preventing falls. The certainty of evidence is judged as low, pointing to the likelihood of change in the evidence in future research. Therefore, future research with robust methodology is needed to elucidate the effectiveness of strategies, either environmental or behavioral or both, designed to increase physical activity and reduce fall risk to reduce the uncertainty prevailing around these strategies. Furthermore, issues regarding the acceptability and feasibility of the interventions need to be considered in future research.

\section{What are the implications of the Cochrane evidence for practice in rehabilitation?}

In older people, visual impairment may cause social isolation, difficulty in walking, and higher risk of falls and fractures with a negative impact on the individual, by limitation of activity and consequently increase in cognitive decline. ${ }^{[2,3]}$ Such increase in morbidity and mortality related to the increased falling incidence calls for more relative research and development of strategies to prevent falls. ${ }^{[5]}$ The strategies need to be both person-directed and also 
take into consideration of the environmental factors such as home environment modification, and attitudes and behaviours as well defined in the International Classification of Functioning, Disability and Health (ICF), which views disability and functioning as outcomes of dynamic interactions between health conditions and environmental and personal factors. ${ }^{[7]}$ Accordingly, Physical Medicine and Rehabilitation physicians could aim at enhancing health behaviors and promoting the favorable influence of contextual factors on functionality of daily living. ${ }^{[8]}$ Therefore, rehabilitation professionals need to be aware of evidence-based environmental and behavioral interventions to implement in rehabilitation programs of care. The results of this Cochrane review ${ }^{[1]}$ provide some evidence of a small benefit of home security modifications in reducing injurious falls based on low certainty evidence. However, the Cochrane review found no conclusive evidence regarding other interventions. The important message here to rehabilitation professionals is that they need to be involved in more research to demonstrate practical results regarding the efficacy of these types of interventions that can be applied to prevent falls and reduce physical activity limitation in older people with visual impairment.

\section{Acknowledgement}

The authors thank Cochrane Rehabilitation and Cochrane Eyes and Vision Group for reviewing the contents of the Cochrane Corner.

\section{Declaration of conflicting interests}

The authors declared no conflicts of interest with respect to the authorship and/or publication of this article.

\section{Funding}

The authors received no financial support for the research and/or authorship of this article.

\section{REFERENCES}

1. E JY, Li T, McInally L, Thomson K, Shahani U, Gray L, Howe TE, Skelton DA. Environmental and behavioural interventions for reducing physical activity limitation and preventing falls in older people with visual impairment. Cochrane Database Syst Rev 2020;9:CD009233.

2. World Health Organization. World report on vision. Geneva: WHO; 2019.

3. Burton MJ, Ramke J, Marques AP, Bourne RRA, Congdon N, Jones I, et al. The Lancet Global Health Commission on Global Eye Health: vision beyond 2020. Lancet Glob Health 2021;9:e489-e551.

4. Hopewell S, Adedire O, Copsey BJ, Boniface GJ, Sherrington C, Clemson L, Close JC, Lamb SE. Multifactorial and multiple component interventions for preventing falls in older people living in the community. Cochrane Database Syst Rev 2018;7:CD012221.

5. James SL, Lucchesi LR, Bisignano C, Castle CD, Dingels ZV, Fox JT, et al. The global burden of falls: global, regional and national estimates of morbidity and mortality from the Global Burden of Disease Study 2017. Inj Prev 2020;26(Supp 1):i3-i11.

6. Yonge AV, Swenor BK, Miller R, Goldhammer V, West SK, Friedman DS, et al. Quantifying Fall-Related Hazards in the Homes of Persons with Glaucoma. Ophthalmology 2017;124:562-71.

7. World Health Organization. International Classification of Functioning, Disability and Health: ICF. Geneva: WHO; 2001.

8. European Physical and Rehabilitation Medicine Bodies Alliance. White Book on Physical and Rehabilitation Medicine (PRM) in Europe. Chapter 1. Definitions and concepts of PRM. Eur J Phys Rehabil Med 2018;54:156-65. 